\title{
Using Polymer Reaction Engineering Principles to Help the Environment: The Case of the Canadian Oil Sands Tailings
}

\author{
João B. P. Soares ${ }^{\circledR *, a}$ and Fernanda L. Motta ${ }^{\circledR a}$ \\ ${ }^{a}$ Department of Chemical and Materials Engineering, University of Alberta, $9211116 \mathrm{St}$, \\ AB T6G 1H9, Edmonton, Canada
}

\begin{abstract}
The Canadian oil sands reserves are an important national resource with a large impact on the Canadian economy. Unfortunately, oil sands extraction processes generate toxic by-products called mature fine tailings that need to be stored in disposal ponds for a very long time before the land can be reclaimed. This serious environmental liability needs to be addressed to reduce the environmental footprint of oil sands operations in Canada. In our research group, we have been applying polymer reaction engineering principles to develop new polymer flocculants specifically designed to treat oil sands tailing ponds. This article gives an overview of the research activities in our laboratory in the area of water-soluble polymer flocculants for the efficient dewatering, consolidation and densification of mature fine tailings.
\end{abstract}

Keywords: oil sands tailings, polymer flocculants, dewatering, densification

\section{Introduction}

After bitumen is extracted from oil sands through the Clark hot water process, the remaining tailings are transported via pipelines to containment ponds. Tailings contain water, coarse sand particles, fine clay and silt particles, and residual bitumen. After being discharged from the pipeline into the disposal area, the coarse particles settle quickly, but about 6 to $10 \mathrm{wt} . \%$ of fine particles remain suspended in the fluid. After a few years of settling, the remaining mixture, called mature fine tailings (MFT), reaches a solids content of 30 to $35 \mathrm{wt} . \%{ }^{1}$ It may take up to 150 years for MFT to consolidate by gravity to the point when the sediments become trafficable and it is possible to reclaim the land occupied by the tailings pond. ${ }^{2}$ The slow consolidation and dewatering of MFT originates from a combination of factors, such as the presence of ultrafine clay particles, ${ }^{3}$ interstratified swellable clays ${ }^{4}$ medium $\mathrm{pH}$ and process water composition. ${ }^{5}$

The Canadian oil sands industry developed rapidly in recent decades, producing a considerable volume of tailings. For over fifty years, MFT has been stored in tailings ponds in northern Alberta. These ponds currently cover about 97 square miles and hold 340 billion gallons of tailings. To put this number in perspective, the volume

*e-mail: jsoares@ualberta.ca of tailings accumulated in these ponds would be enough to fill more than half a million Olympic-size swimming pools. ${ }^{6}$ The volume of tailings ponds continues to grow, forming one of the major environmental challenges associated with Canada's biggest energy play. The pressing question is how to treat this massive volume of tailings and remediate the artificial basins in which they are held.?

Over the last two decades, much effort has been devoted to develop technologies for oil sand tailings treatment. Most of the current technologies rely on the use of commercial polymer flocculants. ${ }^{8}$ Anionic polyacrylamide (PAM) with ultrahigh molecular weight $\left(>10\right.$ million $\left.\mathrm{g} \mathrm{mol}^{-1}\right)$ are widely used to treat industrial and urban wastewaters. Flocculation with PAM, however, forms loosely packed sediments with a gel-like structure that trap water via hydrogen bonding with the polymer chains. ${ }^{8,9}$ The shear strength of the sediments formed with PAM is also low, which represents an obstacle to tailing ponds reclamation because they are not strong enough to become trafficable. ${ }^{10}$

Therefore, we need to design new polymer flocculants that are more effective than the current generation of commercial additives. Our research group has successfully developed a series of multifunctional copolymers, terpolymers and graft polymers to flocculate oil sands tailings. We have also synthesized a library of bio-based flocculants based on chitosan, amylopectin, $\varepsilon$-caprolactone, and lactide, as well as explored thermosensitive, 
pH-sensitive, polyelectrolytes and hydrophobicallymodified polymers. In this paper, besides giving an overview of several polymers developed in our laboratory, we discuss the effect of key flocculation conditions and flocculant properties on MFT dewatering and consolidation behavior.

\section{Potential Alternative Polymer Flocculants for MFT Dewatering}

Currently, no mature technology can effectively treat oil sands tailings to the point that tailings ponds can be completely eliminated. ${ }^{8}$ However, many studies have shown that when tailings are mixed with appropriate flocculants, the sedimentation time is significantly reduced, a relatively high solid content is achieved and the sediments' strength increases. The use of polymer flocculants may also improve the performance of other tailings dewatering technologies such as centrifugation, filtration and evaporation/drying.

Flocculation is a process in which suspended solids gather in larger aggregates via polymer bridging. Since the flocs have higher densities than the surrounding medium, they settle rapidly and produce clear supernatants under optimal conditions. Polymer chains adsorb onto one or more MFT clay particle sites via hydrogen bonding and/or electrostatic interactions. Aggregates formed by flocculation have structures that are more open than those formed by coagulation (which is driven only by charge neutralization) due to the high lengths of the polymer chains. Unfortunately, this open floc structure traps a large amount of water within the aggregates. ${ }^{11}$

Several performance metrics are adopted to assess MFT flocculation and dewatering behavior: $(i)$ sediment volume reduction, determined by measuring the normalized mudline height and initial settling rate (ISR); (ii) ease of further dewatering, quantified by capillary suction time (CST) and specific resistance to filtration (SRF); (iii) quality of supernatant, assessed by measuring supernatant turbidity and (iv) yield strength, which is related to the solids content of the sediments; while thixotropic and temporary viscosity effects may affect apparent yield strength, solids content is an absolute measurement of consolidation. ${ }^{12}$

Mudline height (the interface between the supernatant and sediments) and total fluid level are measured using a graduated cylinder, and used to calculate the normalized mudline height, given as the ratio: mudline height/total fluid level. The ISR is calculated by measuring the slope of the linear portion of the mudline height versus time curve.

Capillary suction time measures the rate of water removal from a sediment sample by capillary action. The test consists of placing a moist sediment on a filter paper and measuring the time taken for the water to move a known radial distance along the paper. The shorter the time required for water to spread, the faster the dewaterability of the samples. Specific resistance to filtration is measured by transferring the slurry into a pressure filtration apparatus. Filtration takes place under applied air pressure for a given period of time, and the mass of released water is recorded at predetermined time intervals. ${ }^{13}$ Values for SRF are calculated using equation $1 .^{14}$

$\mathrm{SRF}=\frac{2 \Delta \mathrm{PA}^{2}}{\mathrm{c} \mu_{\mathrm{f}}} \times \mathrm{b}$

where $\Delta \mathrm{P}$ is the applied pressure in $\mathrm{Pa}, \mathrm{A}$ is the filter medium area, $\mathrm{b}$ is the slope of $\mathrm{t} / \mathrm{V}$ against $\mathrm{V}$ ( $\mathrm{t}$ is time and $\mathrm{V}$ is the volume of the collected water), $\mathrm{c}$ is solid concentration in the slurry, and $\mu_{\mathrm{f}}$ is the filtrate viscosity. Compared with CST, which is simple and inexpensive to operate, SRF measurements are more complicated, time consuming and more expensive to complete. ${ }^{15-17} \mathrm{CST}$ values, however, cannot predict how the physical properties of the sample change during the dewatering process.

Supernatant turbidity is measured using a turbidimeter and expressed in nephelometric turbidity unit (NTU). Finally, the solids content of the sediment is calculated by measuring its mass before and after complete drying and expressed as the ratio (dry mass)/(wet mass).

In flocculation, polymer architecture is very important to control adsorption and subsequent polymer configuration on the solid surfaces. Regarding charged polymers, the optimum charge density would ensure the polymer would have sufficient flexibility to form loops with moderate binding forces. ${ }^{11}$ Various polymers, both natural and synthetic, have been used to flocculate MFT. Biopolymers, mainly polysaccharides, are usually biodegradable, nontoxic and shear-stable. ${ }^{18}$ Another advantage of biopolymers is that they have wide sources and low cost. Compared to natural polymers, however, synthetic polymers are more effective flocculants because their molecular structures can be tailored to meet specific application demands. ${ }^{19}$ Among synthetic polymers, those commonly used comprise poly(ethylene oxide) in the nonionic category, poly(diall yldimethylammoniumchloride) (polyDADMAC) in the cationic category, and PAM and poly(styrenic sulfonic acid) in the anionic category. ${ }^{19}$ Acrylamide-based polymers are widely used as flocculants in wastewater treatment, and oil sands tailings treatment has benefited from using these polymers to a certain extent. ${ }^{11}$

It is not our intention to present an exhaustive review of this complex field in this article. Rather, we will summarize 
some of our recent work, focusing on the development and testing of novel MFT polymer flocculants.

\section{Poly(acrylamide)-graft-polypropylene Oxide}

One of the limitations of conventional PAM flocculants used to treat MFT is that they form flocs that trap water via hydrogen bonding. One approach that may reduce this negative effect is to decrease the hydrophilicity of the backbones by adding hydrophobic groups into PAM. ${ }^{20}$ The rationale behind this approach is that less hydrophilic chains will still behave as effective flocculants, but will form flocs that contain less water and that are also easier to dewater.

By copolymerizing different amounts of polypropylene oxide macromonomers (PPO) with PAM we produced poly(acrylamide)-graft-polypropylene oxide (PAM- $g$-PPO) copolymers with different hydrophobicities and molecular weights (Figure 1). The PAM- $g$-PPO copolymers were less hydrophilic than commercial PAM and were more likely to expel water from the flocculated MFT, reducing the water retained inside the flocs formed during MFT treatment. Furthermore, by changing the frequency and molecular weight of the PPO macromonomers grafted onto the PAM backbones, we could optimize polymer hydrophobicity and flocculation behavior, enhancing dewatering performance while maintaining the solubility of PAM- $g$-PPO in water.

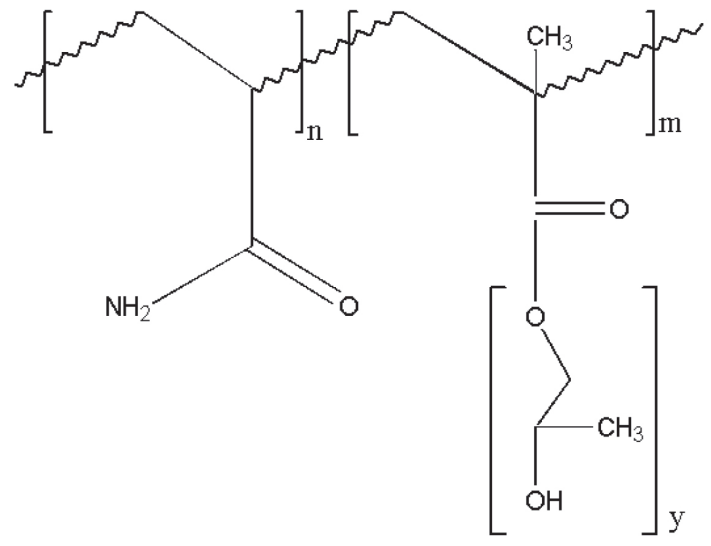

Figure 1. Structure of PAM- $g$-PPO where $n, m$ and y are repeating units of the portion in parentheses (adapted from reference 20).

Besides densifying and dewatering tailings more efficiently, the PAM- $g$-PPO copolymers had molecular weights that were ten times smaller than the reference anionic PAM flocculant, which reduced their solution viscosities and shear-sensitivities. As a result, it was easier to mix these copolymers with tailings under a wider window of operating conditions.

\section{Poly(acrylamide)-graft-poly(ethylene oxide methyl ether methacrylate)}

Inspired by the promising results obtained with the PAM- $g$-PPO flocculants, ${ }^{20}$ we synthesized a series of polyacrylamide-poly(ethylene oxide methyl ether methacrylate) copolymers (PAM- $g$-PEOMA) ${ }^{10}$ (Figure 2). A central composite design of experiments was used to study how the microstructure of PAM- $g$-PEOMA (PEOMA/PAM ratio, molecular weight and PEOMA length) affected the flocculation and dewatering of MFT.

Adding a large fraction of hydrophobic grafts (PEOMA > $30 \mathrm{wt} . \%$ ) enhanced the performance of PAM- $g$-PPO, as measured by CST and ISR, but did not help flocculate most of the fine particles, leading to supernatants with higher turbidities. PAM- $g$-PEOMA with higher molecular weight averages (made using lower free radical initiator concentrations) performed better in most situations. The length of the hydrophobic PEOMA grafts did not have an important effect: PAM- $g$-PEOMA with shorter PEOMA chains present in larger percentage performed similarly to PAM- $g$-PEOMA with longer PEOMA chains present in lower percentage.

Surprisingly, all PAM- $g$-PEOMA copolymers tested were adequate flocculants, even though most of them had relatively low molecular weights $\left(<200,000 \mathrm{~g} \mathrm{~mol}^{-1}\right)$. This is an additional advantage of this family of flocculants, since polymers with lower molecular weights have a wider window of mixing conditions where flocculation can be performed effectively than polymer with higher molecular weights.

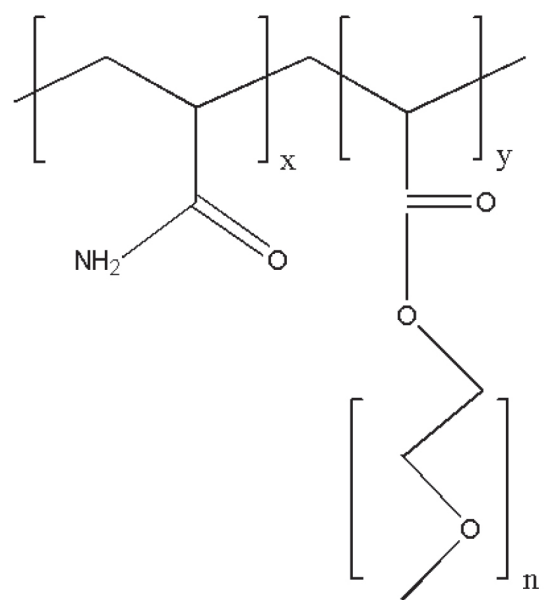

Figure 2. PAM- $g$-PEOMA structure, where $\mathrm{x}, \mathrm{y}$ and $\mathrm{n}$ are repeating units of the portion in parentheses (adapted from reference 10).

\section{Poly(acrylamide-co-diallyldimethylammo- nium Chloride)}

The surfaces of the clay particles suspended in MFT 
are negatively charged. Neutral flocculants interact with these surfaces via van der Waals forces and hydrogen bonding, while anionic flocculants need the help of divalent bridging cations such as $\mathrm{Ca}^{2+}$. Choosing a cationic polymer, or at least a copolymer in which one of the comonomers is cationic, seems to be a logic solution to increase the interaction of the polymer chains with the clay surfaces via electrostatic forces.

Following this lead, we assessed the effect of chemical composition and average molecular weight of poly(acrylamide-co-diallyldimethylammonium chloride) using design of experiments and surface response methodologies (Figure 3). ${ }^{13}$ A central composite design was adopted to assess the flocculation performance of our novel copolymers in terms of ISR, turbidity, CST and SRF. Compared to conventional one-factor-at-a-time approach, the central composite design can find the effect of the studied parameters and their interactions with fewer number of experiments. ${ }^{21,22}$

Copolymer composition and molecular weight significantly affected ISR and supernatant turbidities, while copolymer composition and polymer dosage affected CST and SRF. Interactions between dosage and copolymer composition affected all flocculation metrics (ISR, turbidity, CST and SRF) tested in this investigation. These results suggest that the dominant mechanism when dewatering MFT with poly(acrylamide-co-diallyldimethylammonium chloride) is charge neutralization, an expected (and hoped for) result, since the diallyldimethylammonium chloride comonomer is positively charged and should neutralize the negative charges on the clay surfaces.

The insights gained with this study are important for

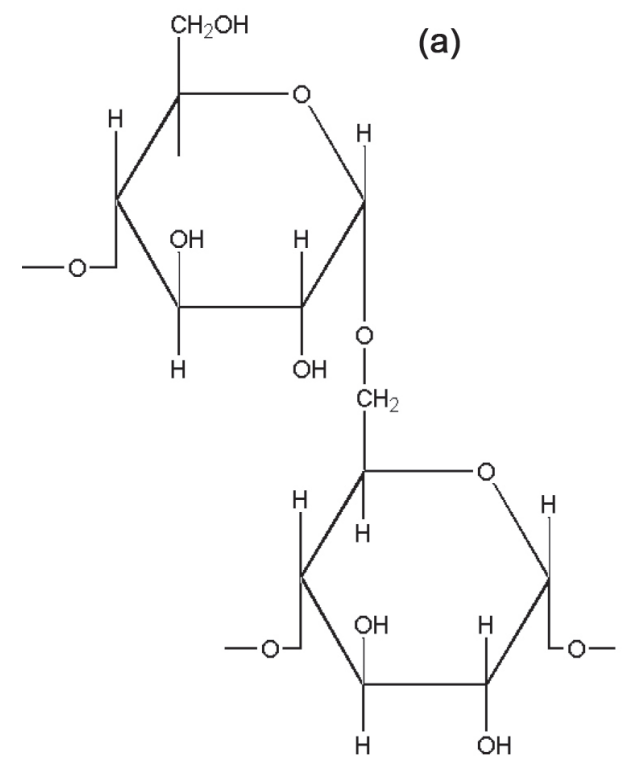

the oil sands industry, which is constantly looking for ways to maximize polymers performance at minimum cost. ${ }^{13}$ In our future studies, we will investigate the effect of the molecular weight distribution of homo- and copolymers on dewatering and densification of MFT.

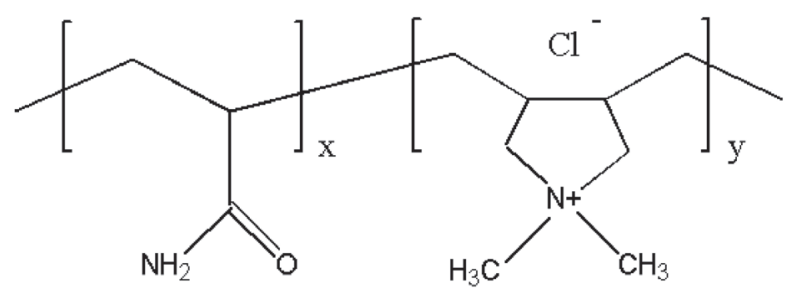

Figure 3. Poly(acrylamide-co-diallyldimethylammonium chloride) structure where $\mathrm{x}$ and $\mathrm{y}$ are repeating units of the portion in parentheses (adapted from reference 13).

\section{Amylopectin-graft-polyacrylamide}

Natural polymers with improved performance by chemical modification have been the subject of extensive research for many decades. In this study, PAM chains were grafted onto amylopectin rigid backbones by free radical polymerization. This novel polymer flocculant, amylopectin- raft-polyacrylamide (AP- $g$-PAM), was evaluated regarding its MFT flocculation and dewatering performance (Figure 4). AP-g-PAM formed flocs with higher solids content than commercial PAM, and AP- $g$-PAM containing longer PAM grafts flocculated MFT better at lower dosages. In addition, flocculation of MFT using AP- $g$-PAM containing longer PAM grafts produced sediments with higher solids content, lower supernatant turbidities, and faster (lower) CST.

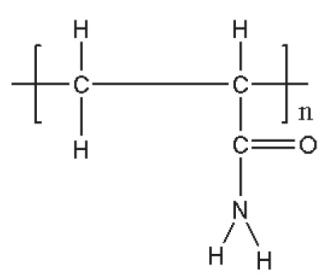

(b)

Figure 4. Chemical structures of amylopectin (a) and PAM (b). 


\section{Chitosan-Based Flocculants}

Although chitosan has been widely used as a flocculant, few papers have studied its use to flocculate MFT. We modified this biopolymer in two different ways: (i) chemical modification with 3-chloro-2-hydroxypropyltrimethylammonium chloride (Chito-CTA) to improve its solubility in water and (ii) PAM grafting (Chito-g-PAM) via free radical polymerization. The dewatering performance of these two flocculants was compared with that of a commercial high molecular weight cationic PAM (Figure 5). ${ }^{23}$

CST measurements showed that Chito-g-PAM could dewater MFT faster than Chito-CTA and the commercial cationic PAM, while no sedimentation was observed when flocculation was performed with the latter. In general, compared to the commercial cationic PAM, the amount of water entrapped in the flocs was lower when MFT was flocculated with our chitosan-based polymers, probably due to the hydrophobic chitosan core. Among the three tested flocculants, Chito- $g$-PAM formed flocs that dewatered at the fastest rates. Flocculation with Chito- $g$-PAM and ChitoCTA resulted in similar SRF values at all tested dosages, while supernatant turbidity was significantly lower for Chito-g-PAM.

Conventionally, calcium ions are used to neutralize the negative charges on MFT clays, facilitating their subsequent bridging by polymeric flocculants. However, calcium adversely affects the quality of the recycled water, ${ }^{24}$ as calcium ions decreases bitumen recovery efficiency. ${ }^{25}$ In this work, no calcium was added to help flocculation, which is desirable in terms of reusing the recovered water from flocculated MFT. Our chitosan-based flocculants are potential candidates to dewater MFT, and additional developments may make these polymers commercially competitive.

\section{Polycaprolactone Choline lodide Ester Methacrylate $\left(\mathrm{PCL}_{\mathrm{n}} \mathrm{ChMA}\right)$}

In this work we produced a new family of cationic flocculants through micellar radical polymerization of cationic polyester macromonomers, polycaprolactone choline iodide ester methacrylate $\left(\mathrm{PCL}_{\mathrm{n}} \mathrm{ChMA}\right)$, which become more hydrophobic in response to hydrolytic degradation. The cationic tips of the comb-like poly $\left(\mathrm{PCL}_{3} \mathrm{ChMA}\right)$ accelerate the settling rate of model tailings containing kaolin (a major component of oil sands tailings). In addition, when subjected to degradation, polyester units are passively degraded by hydrolysis and reveal hydrophobic segments that further expel water from the sediments, reducing CST by about 30\%. When compared to commercial PAM, poly $\left(\mathrm{PCL}_{3} \mathrm{ChMA}\right)$ produced more compact sediments and supernatants with lower turbidities. ${ }^{26}$

Following up the initial promising results with the kaolin-based model tailings, ${ }^{26}$ we investigated the influence of copolymer structure on MFT flocculation behavior. ${ }^{27}$ In order to make a homopolymer with both appreciable cationic density and hydrophobic content, while still maintaining its hydrolytic degradation characteristics, we adjusted the average number of degradable units in the polyester side chain to 2 ( $\mathrm{PCL}_{2} \mathrm{ChMA}$ ). We also investigated the influence of copolymerizing $\mathrm{PCL}_{2} \mathrm{ChMA}$ with PAM. Although by performing the copolymerization we could control polymer charge density and hydrophobicity, higher dosages were needed to achieve the same performance as the corresponding homopolymers. ${ }^{27}$

This study demonstrated that the dewatering efficiency of the novel $\mathrm{PCL}_{\mathrm{n}} \mathrm{ChMA}$ flocculants, specifically designed for MFT treatment, improved upon in situ hydrolytic degradation. Further work is underway to proceed with larger-scale testing. ${ }^{27}$
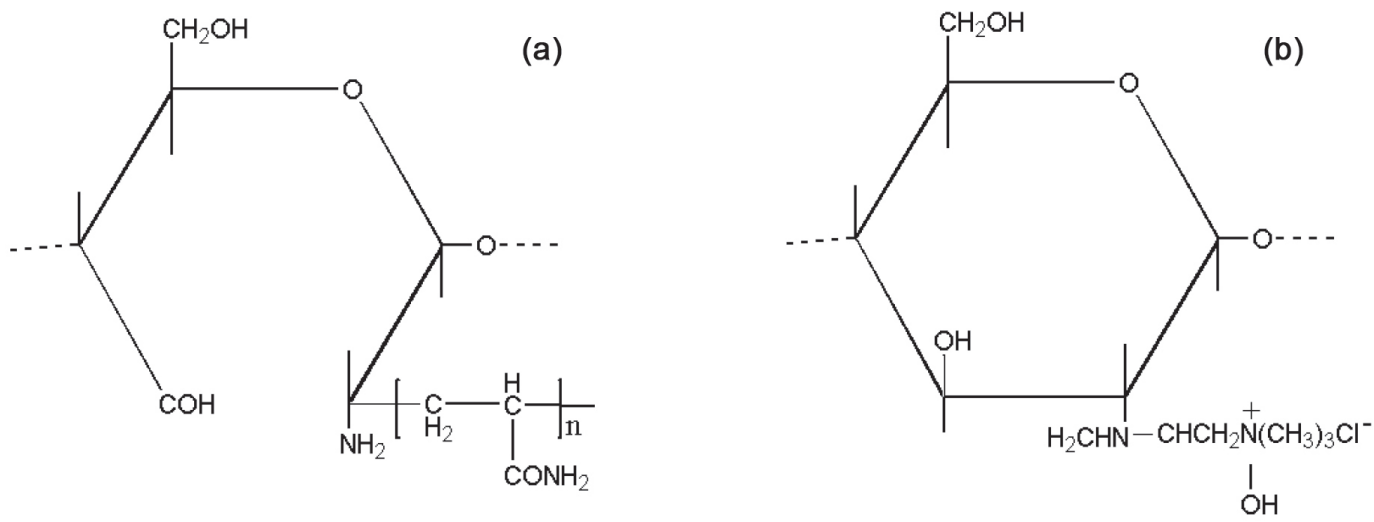

Figure 5. Structures of (a) chitosan grafted with PAM (Chito-g-PAM) and (b) chitosan modified with CTA (Chito-CTA) (adapted from reference 23). 


\section{Poly( $N$-isopropyl acrylamide/acrylic acid/N-tert-butylacrylamide)}

The effect of multifunctional poly( $N$-isopropyl acrylamide/acrylic acid/N-tert-butylacrylamide) [p(NIPAM-AA-NTBA)] ternary polymer (Figure 6) on kaolin suspension (model MFT) sedimentation was investigated in terms of ISR, supernatant turbidity and $\mathrm{CST}^{28}$

Due to the presence of $N$-isopropyl acrylamide (NIPAM), acrylic acid (AA), and $N$-tert-butylacrylamide (NTBA) in its chains, the ternary copolymer flocculant combined comonomers with thermo-sensitivity (NIPAM), anionic charges (AA) and hydrophobic association (NTBA) properties. This thermosensitive ternary polymer undergoes an extended to coil-like conformation transition above its lower critical solution temperature. Therefore, flocculation tests at $50{ }^{\circ} \mathrm{C}$ led to higher ISR than at room temperature.

We examined the effect of number average molecular weight, charge density, and concentration of NTBA on kaolin suspension sedimentation and observed that faster ISR was obtained with polymers with higher molecular weights. Up to a certain extent, adding the anionic comonomer helped increase ISR and lower turbidity. In addition, by incorporating the hydrophobic comonomer, the best flocculation performance in terms of dewaterability, ISR and supernatant turbidity was achieved. Above the optimum fraction of AA, electrostatic repulsion between flocculantcoated particles dominated. Although the presence of the hydrophobic comonomer enhanced dewaterability, if the NTBA fraction in the copolymer was too high, it lowered the solubility of the flocculant in water. The polymer lower critical solution temperature increased when copolymerized with AA and decreased when copolymerized with NTBA. The anionic comonomer, AA, played a vital role in neutralizing the charges onto clay particles, whereas NTBA helped to improve the dewaterability of the sediments, which indicates that optimum fractions of these comonomers are desirable to balance the performance of this complex tertiary polymer flocculant.

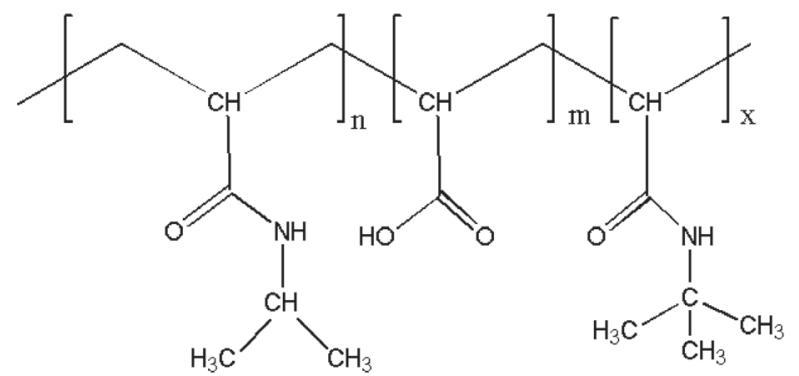

Figure 6. Chemical structure of p(NIPAM-AA-NTBA) (adapted from reference 28).

\section{Hyperbranched Functionalized Poly- ethylenes (HBfPE)}

Our previous studies pointed out that the performance of polymer flocculants for dewatering and densification of MFT may be improved through hydrophobic modification. Not only hydrophobic domains in the polymer chain will trap less water, but it is also possible that they will interact more intimately with the fugitive bitumen (2-5 wt.\%) present on the clay surfaces, presenting new sites for adsorption and bridging the clay particles.

In this study, we functionalized a purely hydrophobic polymer backbone (polyethylene) with charged functional groups contents ranging from 30 and $50 \mathrm{~mol} \%$ to enable polymer dispersal in water and interaction with the clay surfaces. We obtained promising results with these hyperbranched functionalized polyethylenes (HBfPE), which indicates that this innovative concept may be useful to create a novel class of flocculants for MFT treatment (Figure 7). ${ }^{29}$

The produced HBfPE had significantly lower viscosities than typical commercial PAM flocculants and required less mixing energy to be properly dispersed in MFT. In addition, HBfPE performed better for MFT samples with higher solids content $(20 \mathrm{wt} . \%)$ than for the samples with lower solids content (5 wt.\%), obtaining lower CST and supernatant turbidities, higher ISR and more compact sediments than a reference PAM. Based on the CST measurements, the sediments generated by $\mathrm{HB} f \mathrm{PE}$ were expected to dewater faster than those formed with PAM. When also combined with centrifugation, the HBfPE flocculants generated sediments with higher solids contents than PAM in shorter centrifugation times. This may be attributed to the tendency of HBfPE to form smaller flocs that compact more tightly than those made with PAM. Within the produced HBfPE series, polymers containing higher functional contents led to better CST and supernatant turbidities, which may be attributed to the formation of more uniform floc sizes.

The next steps of this work in our group will include scale up of the polymer synthesis and evaluation of its long-term dewatering performance and saturated hydraulic conductivities. $^{29}$

\section{Amylopectin Grafted Hydrolyzed Poly(methyl acrylate) (AP-g-H-PMA)}

In this study, poly(methyl acrylate), a polymer known to create small flocs and efficiently capture both large and fine particles when hydrolyzed, was used to synthesize amylopectin grafted hydrolyzed poly(methyl acrylate) 


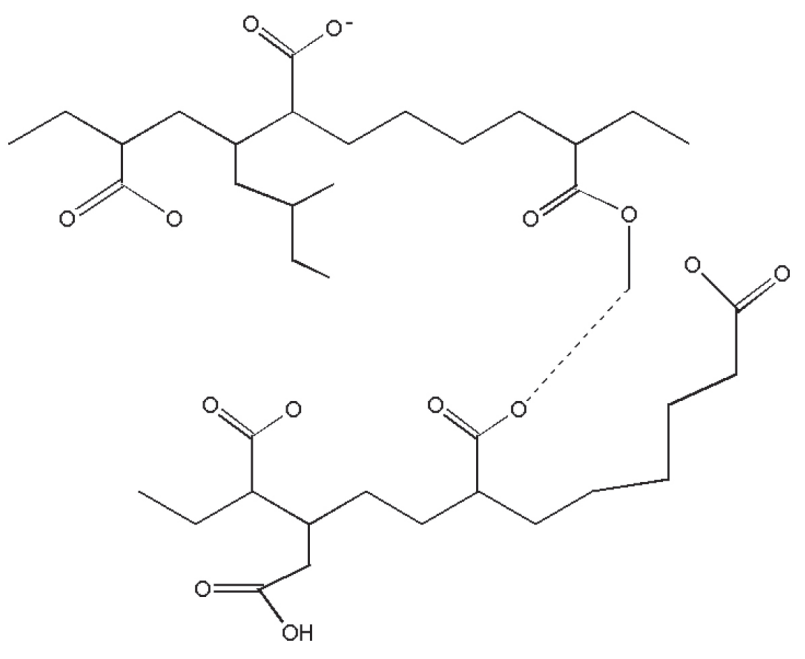

Figure 7. Structure of HBfPE (adapted from reference 29).

(AP-g-H-PMA). The flocculation performance of this grafted polymer was compared to amylopectin, hydrolyzed poly(methyl acrylate), and the combined homopolymers using various flocculation metrics including ISR, CST, solids content and turbidity. A dosage study showed that good performance is achievable within a wide polymer dosage range (500-2000 ppm solids basis). It is important to note that despite the grafted polymer performing the best overall, the effect of dosage changed the results of each metric test. The ISR and CST at higher dosages obtained similar or slightly better results than the commercial reference polymers. However, the solids content and turbidity of the grafted polymer at lower dosages outperformed the separated and combined homopolymers.

As it is known that the flocculation performance of polymers strongly depend on the mixing condition especially in large-scale experiments, we studied the effect of scaling up on flocculation performance of our AP- $g$-H-PMA. Preliminary results showed that higher dosages of polymer are needed when scaling up the experiments. The scale up tests resulted in large variations of CST, solids content and turbidities, which indicates that the results from lab scale tests are no reproducible in large scale experiments and flocculation performance of a polymer might be completely different when testing in large scales, and emphasizes the importance of the studies on scaling up the mixing process.

\section{Polymer Microstructure-Dewatering Relationship and Effect of Operational Conditions}

Solid-liquid separation efficiency in oil sands tailings is largely affected not only by the polymer microstructure (i.e., molecular weight, composition, charge, etc.), but also on a number of factors such as properties of MFT (solids content, particle size, particle surface property, solution chemistry), polymer dosage, temperature, mixing conditions and flow rate. In addition, dilution factor and viscosity of polymer solution also play an important role on the performance of flocculants. These variables affect the floc evolution in time, mode of flocculation, aggregate size, density, dewaterability, compressibility, among others.

Due to their high molecular weight, polymer flocculants usually form very viscous stock solutions. Proper mixing is required to disperse such solutions uniformly into suspension rapidly, and to induce collisions between particles and polymer molecules. Inadequate mixing causes a local overdose of the polymers, resulting in steric stabilization which is thought to be the origin of residual haze and poor filterability in flocculated suspensions, ${ }^{30}$ also it does not allow flocs to grow sufficiently large in the time period available. On the other hand, excessive agitation causes breakage of flocs, leading to a reduction of possible maximum floc sizes. ${ }^{31}$ It is interesting to note that some studies indicated that certain degree of floc breakage can in fact improve the dewaterability of MFT. ${ }^{32}$ Clearly, for each individual system, there appears to be an optimum mixing scheme (time and intensity). ${ }^{33,34}$

The type of charge and charge density of the polymer also have a significant effect on its interaction with particles. Firstly, the conformation of polymers in solution depends on their charge. Anionic and cationic polymers, due to the electrostatic repulsion between charged segments, have a more extended conformation than a neutral polymer. This extended conformation leads to more effective bridging and favors flocculation, as it increases the hydrodynamic radius of the polymer. Furthermore, electrostatic interactions between particles and polymer significantly affect adsorption rates, increasing it in oppositely charged polymer-particle systems, and inhibiting it in systems where polymer chains and dispersed particles have the same charge, especially for low molecular weight, high charge-density polymers. For high molecular weight, low charge-density polymers, interactions among their uncharged segments and the surface of the suspended particles may be strong enough that the electrostatic effects become less important. ${ }^{32}$

In general, high molecular weight polymers are needed in bridging flocculation, as the molecule must be large enough to span the gap between particles and extend beyond the range of electrostatic repulsion. The adsorbed polymer also increases the effective hydrodynamic radius of the particle allowing collisions to occur more readily; therefore a greater molecular weight is beneficial. ${ }^{35}$ Furthermore, high 
molecular weight flocculants can form more links with the particle surface than can low molecular weight flocculants, leading to stronger flocs. ${ }^{36}$ However, when treating MFT, polymers with lower molecular weights are easier to be dispersed due to their lower solution viscosities. In addition, polymers with lower molecular weights are less shearsensitive than their high molecular weight counterparts, widening the window of mixing conditions where optimum flocculation and dewatering can be achieved. ${ }^{20}$

Since cations may also have a substantial effect on clay coagulation and polymer conformation and performance, the water chemistry of MFT is critical for their sedimentation/flocculation behavior. ${ }^{37}$ The medium $\mathrm{pH}$ determines the suspended particles charge and may also affect the polymer flocculant conformation. ${ }^{32}$ Another parameter that affects MFT flocculation is the particles size distribution of the suspended clays. MFT generally contains particles smaller than $44 \mu \mathrm{m}$ (silts and clays), with most particles being approximately $22 \mu \mathrm{m}$ or smaller. ${ }^{38}$ As smaller particles have higher surface charges, the repulsion and the distance between them are also higher, and more energy is needed to aggregate them. Therefore, smaller particles form stable suspensions that are difficult to flocculate. ${ }^{39}$

Flocculation parameters need to be optimized for every new polymer used, which requires intensive laborious work. Mathematical models describing the flocculation behavior in tailings in presence of polymeric flocculants can be very helpful to reduce the laborious optimization. A modeldriven approach to predict which flocculant microstructure will achieve the desired set of performance criteria at given process conditions needs to be established. Further, various experiments or reliable literature data can "train the model" to effectively predict the output parameters. ${ }^{11}$ We are interested in developing mathematical models to predict flocculation process in oil sands tailings treatment. Population balance models are generally used to describe change in the size and distribution of particles or aggregates in a system. We monitor the flocculation experiments by the focused beam reflectance measurement and use these experimental results to train and validate our model. We recently used a population balance model to describe the flocculation of MFT. A time varying function was defined to account for the aggregate size evolution during MFT flocculation induced by polymer chains relaxation and rearrangement on the particle surfaces. By using focused beam reflectance measurement and varying the mixing time, flocculant dosage and shear rate, we tested the validity of the model, which is a first step towards a more systematic approach to evaluate flocculation of MFT. ${ }^{40}$

\section{Conclusions}

The search for the "ideal" flocculant for the treatment of oil sands tailings is continuing. Oil sands tailings are complex systems comprising sand, clays of different types, fugitive bitumen in different proportions and varying water chemistry. Since tailings vary from source to source, as well as a function of their age in the ponds, flocculants that perform well for one given tailing type may not show the same performance for a sample collected from a different source. It is unlikely that we will find the proverbial "silver bullet" that will be able to treat all tailings effectively. It is also probably unlikely that the final solution for tailing ponds can be reached using only polymer flocculants. More likely, it will require the combination of a polymer flocculant specifically formulated for a particular tailing type in combination with supplementary physical purification means such as filtration, centrifugation, drying, or thin lifting.

It is apparent that one needs to understand how the polymer architecture affects its performance as a flocculant for oil sands tailings. We know that a certain degree of hydrophilicity is needed, since the polymer must dissolve in the aqueous medium of the tailings, but adding some hydrophobic groups or grafts helps dewatering of the sediments, and may also enhance interactions with fugitive bitumen present in the clays. High polymer molecular weights (in reality, hydrodynamic radius) is a must to enhance the bridging mechanism, but after a certain point it becomes a liability because it makes the flocculant to become shear sensitive, initially forming large flocs that can be broken under shear stresses as the tailings are transported from the extraction sites to their final destinations in the tailing ponds. It is also clear that cationic comonomers may help enhance floc formation by electrostatic interaction with the negatively-charged surfaces of the clays, but after a certain ionic charge they may "wrap" around the clay surfaces and fail to act as bridges, rather becoming simple coagulants.

Quantitatively, some of these phenomena are understood, or at least intuited. Unfortunately, there is a lack of quantitative information on how to tailor each of these properties to make polymer flocculants with optimal performance for a particular type of oil sands tailings and tailing remediation process. The road ahead of us still seems long, but we are starting to understand its building blocks. Polymer reaction engineering fundamentals and rigorous statistical analysis of multivariate data will, not doubt, play an essential role in solving this pressing environmental problem. 


\section{Acknowledgments}

Most of the work reviewed in this article has been supported by the Campus Alberta Innovates Program (CAIP). We also would like to acknowledge the hard work and dedication of a large number of post-doctoral fellows, professors, graduate and undergraduate students who have spent many hours working on these new polymer flocculants: Behnaz Bazoubandi, Vahid Vajihinejad, Sarang P. Gumfekar, Marco da Silva, Linda Botha, Leidiane G. Reis, Roberta S. Oliveira, Thiago N. Palhares, Luciana S. Spinelli, Elizabete F. Lucas, Diógenes R. L. Vedoy, Elliott Asare, Rhys Hripko, Rina Guillermo, Leonardo Pennetta de Oliveira, Thomas R. Rooney, Robin A. Hutchinson, Stepheney Davey, Benjamin Nguyen, Anindya K. Swarnakar, Eric Rivard. Some of the results discussed in this paper are part of unpublished thesis work of students listed above.

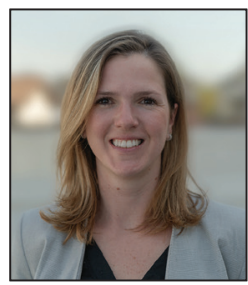

Dr Fernanda Lopes Motta received her bachelor's and master's degree in Food Engineering from the University of Viçosa (Brazil) and University of Campinas (Brazil), respectively. She did her $P h D$ in Chemical Engineering at the University of Campinas and worked as a postdoctoral fellow under the supervision of Prof João Soares at the University of Alberta (Canada). She is currently a research scientist at Natural Resources Canada (NRCan) and an Adjunct Professor in the Department of Chemical and Materials Engineering at the University of Alberta. She is especially interested in polymer synthesis and engineering, fermentation and water treatment.

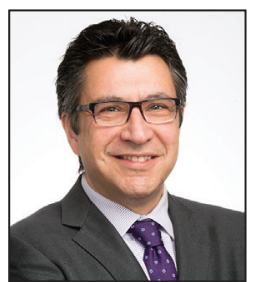

Dr João Soares is a Professor in the Department of Chemical and Materials Engineering at the University of Alberta. He holds a Campus Alberta Innovates Program Chair in Interfacial Polymer Engineering for Oil Sands Processing, and a Tier I Canada Research Chair in Advanced Polymer Reaction Engineering. He is the Editor-in-Chief for the Canadian Journal of Chemical Engineering, a Fellow of the Chemical Institute of Canada and of the Canadian Academy of Engineering, and a Professional Engineer in the Provinces of Ontario and Alberta. He is a specialist in polymerization reaction engineering and polymer characterization.

\section{References}

1. https://www.cosia.ca/uploads/files/Media\%20Resources/ Media\%20Kit/Preventing\%20the\%20Production\%20of\%20 Fluid\%20Fine\%20Tailings.pdf, accessed in June 2018.

2. Fedorak, P. M.; Coy, D. L.; Salloum, M. J.; Dudas, M. J.; Can. J. Microbiol. 2002, 48, 21.

3. Mercier, P. H. J.; Ng, S.; Moran, K.; Sparks, B. D.; Kingston, D.; Kotlyar, L. S.; Kung, J.; Woods, J.; Patarachao, B.; McCracken, T.; Pet. Sci. Technol. 2012, 30, 915.

4. Omotoso, O. E.; Mikula, R. J.; Appl. Clay Sci. 2004, 25, 37.

5. MacKinnon, M. D.; Matthews, J. G.; Shaw, W. H.; Cuddy, R. G.; Int. J. Surf. Min., Reclam. Environ. 2001, 15, 235.

6. http://calgaryherald.com/business/energy/the-battle-over-whenand-how-to-clean-up-oilsands-tailing-ponds-is-escalating, accessed in June 2018.

7. http://calgaryherald.com/business/energy/tailings-ponds-acritical-part-of-albertas-oilsands-legacy, accessed in June 2018.

8. Vedoy, D. R. L.; Soares, J. B. P.; Can. J. Chem. Eng. 2015, 93, 888.

9. Cong, R.; Pelton, R.; J. Colloid Interface Sci. 2003, 261, 65.

10. Hripko, R.; Vajihinejad, V.; Lopes Motta, F.; Soares, J. B. P.; Global Challenges 2018, 2, 1700135.

11. Gumfekar, S. P.; Soares, J. B. P.; Chemosphere 2018, 210, 156.

12. Thompson, D. K.; Motta, F. L.; Soares, J. B. P.; Miner. Eng. 2017, 111, 90 .

13. Vajihinejad, V.; Guillermo, R.; Soares, J. B. P.; Ind. Eng. Chem. Res. 2017, 56, 1256.

14. Alamgir, A.; Harbottle, D.; Masliyah, J.; Xu, Z.; Chem. Eng. Sci. 2012, 80, 91.

15. Ayol, A.; Process Biochem. 2005, 40, 2427.

16. Teoh, S.-K.; Tan, R. B. H.; Tien, C.; Chem. Eng. Sci. 2006, 61, 4957.

17. Yukseler, H.; Tosun, İ; Yetis, U.; J. Membr. Sci. 2007, 303, 72.

18. Singh, R. P.; Pal, S.; Krishnamoorthy, S.; Adhikary, P.; Ali Sk, A.; Pure Appl. Chem. 2009, 81, 525.

19. Krishnamoorthi, S.; Singh, R. P.; J. Appl. Polym. Sci. 2006, 101, 2109.

20. Reis, L. G.; Oliveira, R. S.; Palhares, T. N.; Spinelli, L. S.; Lucas, E. F.; Vedoy, D. R. L.; Asare, E.; Soares, J. B. P.; Miner. Eng. 2016, 95, 29.

21. Czitrom, V.; Am. Stat. 1999, 53, 126.

22. Neter, J.; Wasserman, W.; Kutner, M. H.; Applied Linear Statistical Models: Regression, Analysis of Variance, and Experimental Designs, $3^{\text {rd }}$ ed.; McGraw-Hill/Irwin: Burr Ridge, 1990.

23. de Oliveira, L. P.; Gumfekar, S. P.; Motta, F. L.; Soares, J. B. P.; Energy Fuels 2018, 32, 5271.

24. https://www.onepetro.org/conference-paper/NACE-03065, accessed in June 2018.

25. Allen, E. W.; J. Environ. Eng. Sci. 2008, 7, 123. 
26. Rooney, T. R.; Gumfekar, S. P.; Soares, J. B. P.; Hutchinson, R. A.; Macromol. Mater. Eng. 2016, 301, 1248.

27. Gumfekar, S. P.; Rooney, T. R.; Hutchinson, R. A.; Soares, J. B. P.; ACS Appl. Mater. Interfaces 2017, 9, 36290.

28. Gumfekar, S. P.; Soares, J. B. P.; Chemosphere 2018, 194, 422.

29. Botha, L.; Davey, S.; Nguyen, B.; Swarnakar, A. K.; Rivard, E.; Soares, J. B. P.; Miner. Eng. 2017, 108, 71.

30. Slater, R. W.; Kitchener, J. A.; Discuss. Faraday Soc. 1966, 42, 267.

31. McFarlane, A.; Yeap, K. Y.; Bremmell, K.; Addai-Mensah, J.; Colloids Surf., A 2008, 317, 39.

32. Klein, C.; Harbottle, D.; Alagha, L.; Xu, Z.; Can. J. Chem. Eng. 2013, 91, 1427.

33. Sworska, A.; Laskowski, J. S.; Cymerman, G.; Int. J. Miner. Process. 2000, 60, 153.
34. Blanco, A.; Negro, C.; Fuente, E.; Tijero, J.; Ind. Eng. Chem. Res. 2005, 44, 9105.

35. Adachi, Y.; Adv. Colloid Interface Sci. 1995, 56, 1.

36. Hogg, R.; Colloids Surf., A 1999, 146, 253.

37. Pourmohammadbagher, A.; Shaw, J. M.; Energy Fuels 2016, 30, 5964.

38. Botha, L.; Soares, J. B. P.; Can. J. Chem. Eng. 2015, 93, 1514.

39. Jewell, R. J.; Fourie, A. B.; Paste and Thickened Tailings: A Guide, $2^{\text {nd }}$ ed.; Australian Centre for Geomechanics, The University of Western Australia: Perth, 2006.

40. Vajihinejad, V.; Soares, J. B. P.; Chem. Eng. J. 2018, 346, 447.

Submitted: August 2, 2018

Published online: October 3, 2018 